(RESEARCH ARTICLE)

\title{
Syndromic osteosarcoma, does it carry a poor prognosis? King Hussein Cancer Center experience. A case series report with literature review
}

\author{
Abdulqader Al-Hebshi ${ }^{1, *}$ and Taleb Ismael 2 \\ ${ }^{1}$ Hematology \& Oncology Division ,Department of Pediatric, Prince Mohammad bin Abdulaziz Hospital (PMBAH), \\ Ministry of National Guard-Health Affairs (MNG-HA), Medina, Saudi Arabia. \\ ${ }^{2}$ Hematology Oncology Division ,Department of Pediatric, King Hussein Cancer Center (KHCC), Amman, Jordan.
}

Publication history: Received on 03 May 2020; revised on 16 May 2020; accepted on 18 May 2020

Article DOI: https://doi.org/10.30574/wjarr.2020.6.2.0141

\begin{abstract}
Osteosarcoma is a tumor derived from mesenchymal cells. Osteosarcoma mainly affects children of 10-14 years old, usually of primary aetiology. Some genetic disorders have been shown to increase osteosarcoma incidence. The aim of our study was to investigate the association of osteosarcoma with syndromic features and the potential effects in the prognosis of osteosarcoma. A case series report of six cases with different syndromes was reported in this study and we have retrospectively evaluated the prognosis of the cases. Data was collected from January 2003 till December 2011 at King Hussein Cancer Center in Jordan. A total of 69 patients were diagnosed to have osteosarcoma during the studied period, 6 of them having associated syndromes, constituting $8.7 \%$ of all cases. The syndromes identified were Rothmund-Thomson Syndrome, Osteogenesis Imperfecta, Diamond-Blackfan Anemia, Osteopoikilosis, and Cockayne Syndrome. From the 63 non-syndromic patients follow up was lost in 14 of them. Among the remaining 49 patients, 34 $(69.3 \%)$ were alive and $15(30.6 \%)$ died, while among the syndromic patients one was lost for follow up, only one remained alive (20\%), and 4 died (80\%). The statistical analysis indicated that syndromic patients presented a poorer prognosis than non-syndromic patients since an association with a higher mortality rate was observed $(\mathrm{p}<0.05) .0$ ur results suggest that syndromic patient's show a poorer prognosis and osteosarcoma should be suspected during differential diagnosis and follow up of their syndromes. Early diagnosis and treatment might improve the outcome and survival.
\end{abstract}

Keywords: Osteosarcoma; Rothmund Thomson Syndrome; Osteopoikilosis; Osteogenesis imperfecta; DiamondBlackfan Anemia.

\section{Introduction}

Osteosarcoma is a tumor derived from mesenchymal cells that can be a consequence of primary causes (without any previous disease) or secondary causes (related to other pathologies). However, the aetiology of osteosarcoma is still partially known and has been described as multifactorial, including genetic alterations, environmental influence, and association with other diseases [1]. Some genetic disorders have been shown to increase osteosarcoma incidence, such as hereditary retinoblastoma, Li-Fraumeni syndrome, Rothmund-Thomson syndrome (RTS), Bloom syndrome, and Werner syndrome [1].Osteosarcoma affects mainly children of 10-14 years old, usually of primary aetiology, and the incidence rate is 4 in 1 million among children of 0-14 years old children [2]. Osteosarcoma is the eighth cause of cancer in children and is the most common primary bone malignancy in children [3]. Furthermore, the incidence of osteosarcoma increases after 65 years old when it is usually originated by secondary causes, such as radiation exposure. Diagnosis is performed through physical examination, laboratory tests, such as alkaline phosphatase and lactate dehydrogenase, imaging diagnosis, and biopsy. After diagnosis, osteosarcoma is classified according to the Enneking system for staging malignant musculoskeletal tumors and American Joint Committee on Cancer system for bone sarcomas $[4,5]$.

\footnotetext{
* Corresponding author: Abdulqader Al Hebshi
} 
Treatment for osteosarcoma includes systemic chemotherapy and local control surgery leading to over $70 \%$ survival rate, but if metastasis or poor responses to treatment are present, prognosis lowers significantly to around $20 \%$ survival rate [6]. In addition, limb amputation may be necessary in some cases. Chemotherapy, surgical intervention, and imaging advances have allowed improving limb salvage. However, the therapeutic approach that had initially raised the survival rate showed no further improvements in the recent years.

An association has been suggested between osteosarcoma and certain syndromes like RTS and Diamond-Blackfan Anemia (DBA) among others, however the incidence is not well known and previous reports indicate different rates. The aim of our study was to investigate the association of osteosarcoma with syndromic features and the potential effects in the prognosis of osteosarcoma. Therefore, a case series report of six cases with different syndromes was reported in this study and we have retrospectively evaluated the prognosis of the cases.

\section{Material and methods}

This is a retrospective study. Data was collected in the period January 2003 till December 2011 at King Hussein Cancer Center in Jordan (KHCC). Ethics Committee Approval was taken prior starting the study. Inclusion criteria covered syndromic and non-syndromic patients diagnosed of osteosarcoma. Information regarding presence of syndromic features, current condition of the patient whether alive, died, or lost, and localized or metastatic disease at diagnosis was retrieved.

\section{Results}

A total of 69 patients were diagnosed to have osteosarcoma during the studied period and 6 of them presented syndromes, constituting $8.7 \%$ of all cases (Table 1). From the 63 non-syndromic patients follow up was lost in 14 of them. Among the remaining 49 patients, 34 (69.3\%) were alive and 15 (30.6\%) died. From the syndromic patients, one was lost for follow up while only one remained alive (20\%) and 4 died (80\%) (Figure 1). The comparison between metastasis percentage in non-syndromic patients vs. syndromic patients indicated that there was no association between metastasis and syndrome presentation $(p>0.05,0 R=3.7)$. However, among mortality rates, syndromic patients presented an association to a higher mortality rate as compared to non-syndromic patients $(\mathrm{p}<0.05,0 \mathrm{R}=9.1)$.

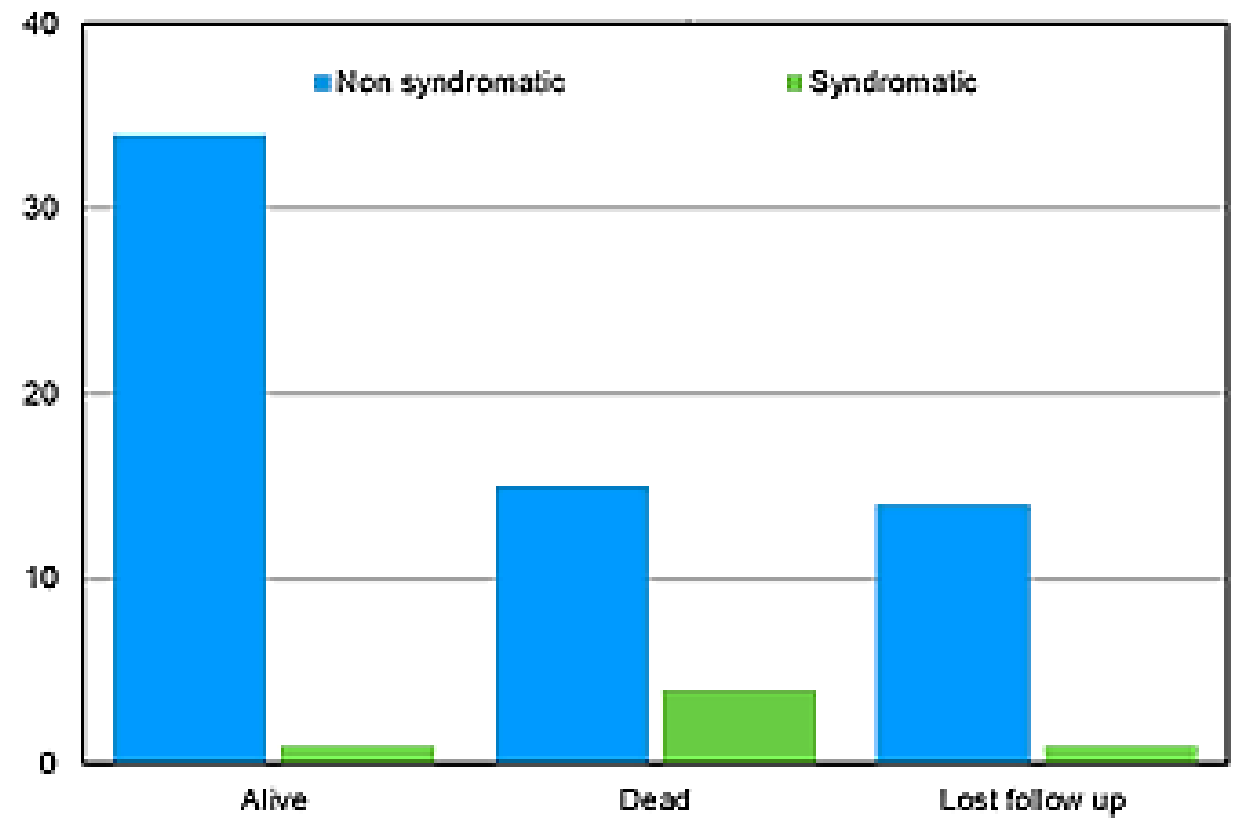

Figure 1 Outcome of the osteosarcoma patients at the end of the study 
Table 1 Distribution of osteosarcoma patients according to their underlying syndromes

\begin{tabular}{|l|l|l|}
\hline Syndrome & N & $\%$ \\
\hline RTS & 2 & 33.3 \\
\hline DBA & 1 & 16.7 \\
\hline Cockayne syndrome (CS) & 1 & 16.7 \\
\hline Osteogenesis Imperfecta (OI) & 1 & 16.7 \\
\hline Osteopoikilosis (OPK) & 1 & 16.7 \\
\hline
\end{tabular}

Abbreviations: DBA: Diamond-Blackfan Anemia; RTS: Rothmund-Thomson Syndrome.

\subsection{Case series report}

\subsubsection{Case 1: Rothmund-Thomson Syndrome}

An 11-year-old boy presented with history of 3 months of occasional left knee moderate pain, which spontaneously disappeared. He experienced a more severe pain one week prior to presentation at KHCC, 4 days after which he exhibited knee swelling and limp while walking. The patient showed antecedents of poor school performance. His family history revealed that his parents were first degree relatives, and his siblings ( 3 sisters and 1 brother) were all healthy. No family history of malignancy was recorded. On physical examination his vital signs were heart rate of $110 \mathrm{bpm}$, temperature of $37.2^{\circ} \mathrm{C}$, and blood pressure of $117 / 70 \mathrm{mmHg}$. His height was $131 \mathrm{~cm}$ on third percentile, indicating that the patient presented a short size. He had a triangular face, telangiectasia on the ear pinna, hyperpigmentation of the skin of ears and face, and nail changes (Figure 2).
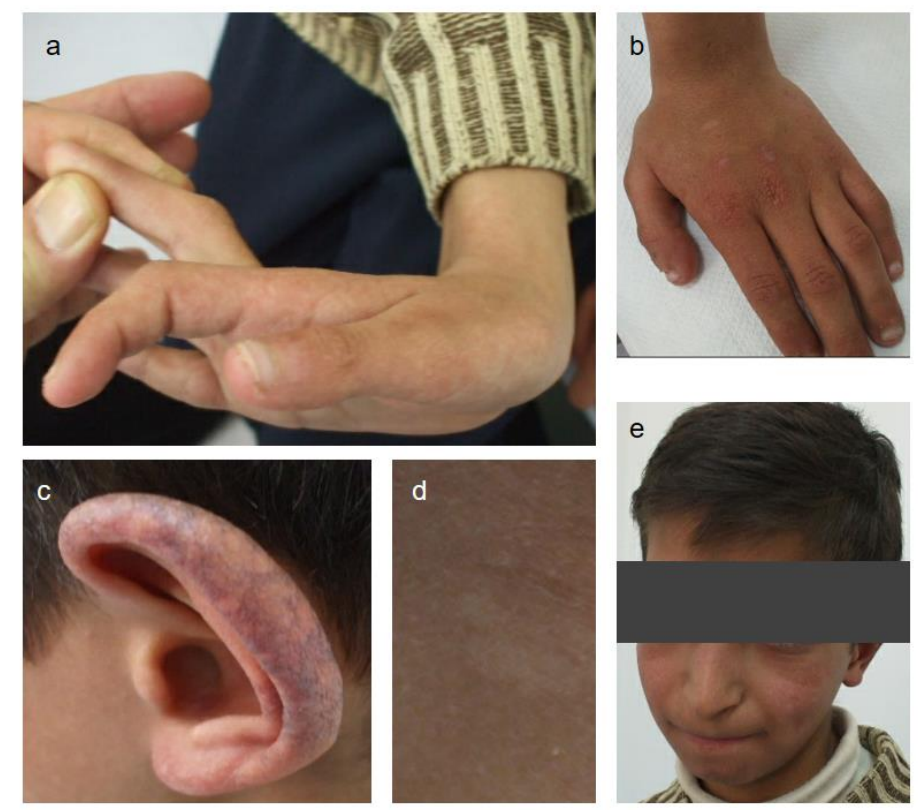

Figure 2 Skin manifestations of RTS clinical case; a and b) nail changes; c) telangiectasia in the ear pinna; d and e) hyperpigmentation of the skin. (Abbreviations: RTS: Rothmund-Thomson syndrome).

There was evidence of swelling on the lateral left thigh. Magnetic resonance imaging (MRI) investigation revealed a large heterogeneous partially sclerotic intramedullary lesion comprising practically the entire length of left femur, extending from the distal femoral metaphysis up to femoral head associated with soft tissue competent at the distal 
femur measuring about $4 \times 5 \mathrm{~cm}$ in transverse dimension (Figure 3). There was no evidence of metastatic disease on chest computed tomography (CT) and bone scan was negative. Pathology confirmed a high grade osteoblastic osteosarcoma. The patient relapsed and his malignancy progressed in lung. After 1.3 years, the patient deceased.
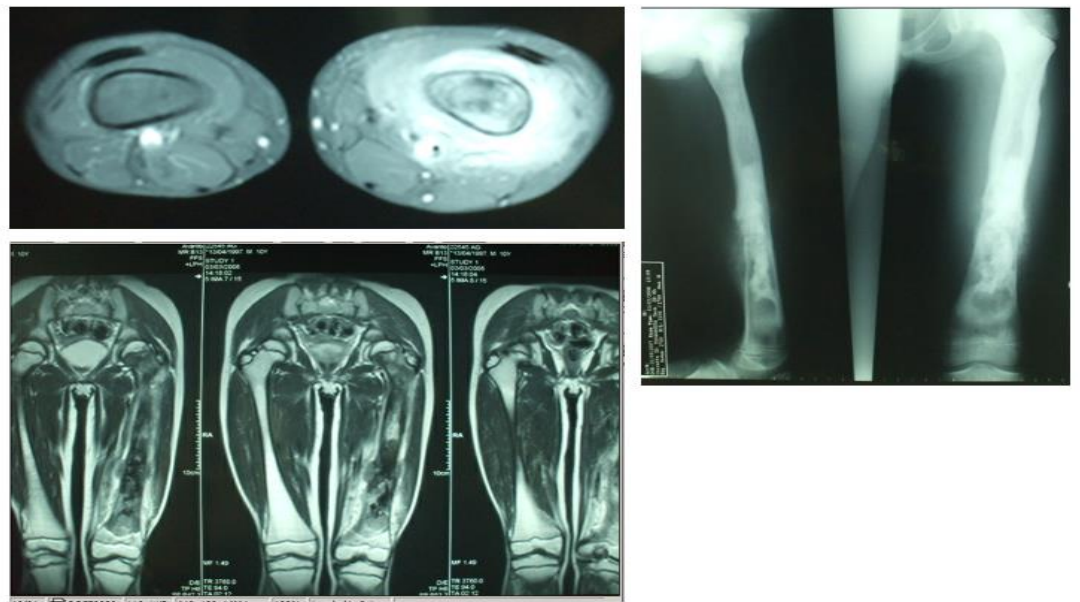

Figure 2 MRI showing a sclerotic intramedullary lesion in the left femur. (Abbreviations: MRI: Magnetic resonance imaging).

\subsubsection{Case 2: Rothmund-Thomson Syndrome}

An 18-year-oldgirl was initially diagnosed in February 1998 with osteosarcoma of the distal part of the left femur at the age of 9 years. Biopsy and tumor resection without amputation were performed in Medical City in Jordan and Palestine. Tumor resection showed positive margins and amputation was carried out in November 1998. Chemotherapy was administered at our hospital KHCC. The patient is alive to the date, after 10 years from treatment with chemotherapy and amputation (Figure 4).
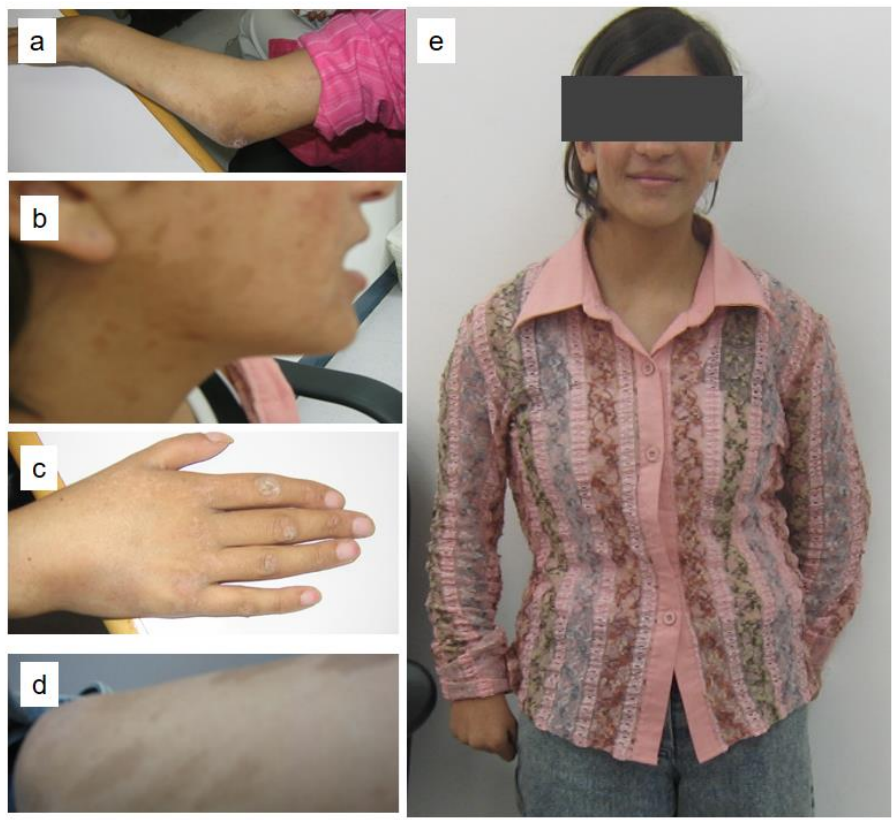

Figure 3 Skin manifestations of a RTS patient;a) and c) poikiloderma in arms and hands;b) and d) hyperpigmentation of the face and skin. (Abbreviations: RTS: Rothmund-Thomson syndrome).

\subsubsection{Case 3: Osteogenesis Imperfecta}

A known case of OI of a 15-year-old girl from Iraq presented with history of multiple fractures. She was treated for left forearm non-metastatic osteosarcoma in 2000 by chemotherapy (5X cisplatin + doxorubicin) and amputation. Her 
family history revealed that 2 brothers died from malignant tumor of bone. There was history of bilateral lower tibia and fibula fractures for which internal plating was done to fix a right tibia fracture, and was removed in March 2009. Six months before the date of arriving the hospital she developed pain and soft tissue swelling at the right ankle. On October $3^{\text {rd }}$ of 2009 biopsy showed spindle cell soft tissue sarcoma. Pathology review at KHCC showed features consistent with high grade osteosarcoma. Her first visit at KHCC was on October $18^{\text {th }} 2009$. On examination her weight was $22.5 \mathrm{~kg}$ and her height of $132 \mathrm{~cm}$ (both below fifth percentile). The sclera conserved their white colour. The patient presented limp while walking with left forearm prosthesis. Presence of lower limbs deformities and scars were also observed. Bone scan showed active bone lesion of the right humerus. Ankle/bone CT revealed a large soft tissue mass likely arising from distal fibula (Figure 5). The patient completed treatment with chemotherapy and amputation, but the follow up was lost after one year post treatment.

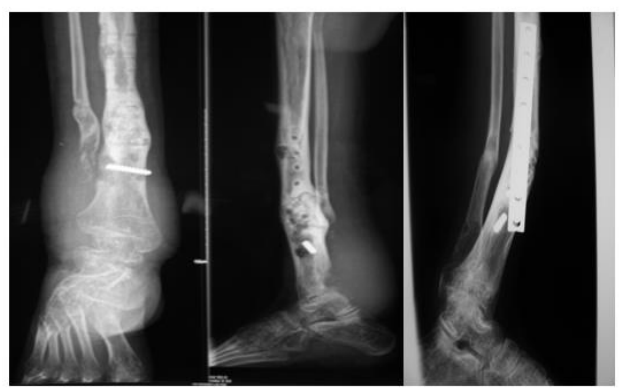

Figure 4 Ankle/bone X-ray revealing a soft tissue mass arising from distal fibula.

\subsubsection{Case 4: Osteopoikilosis}

A 17-year-old boy presented with right upper tibia swelling of four months duration, with previous diagnosis outside KHCC of right proximal tibia osteosarcoma (Figure 6). He presented low weight at birth with no complications. At the age of 2 years there was growth retardation and mild mental retardation, hearing loss with erythematous skin rash with scaling and bleeding following sun exposure. He progressed to lung relapse and died after 1.8 years.
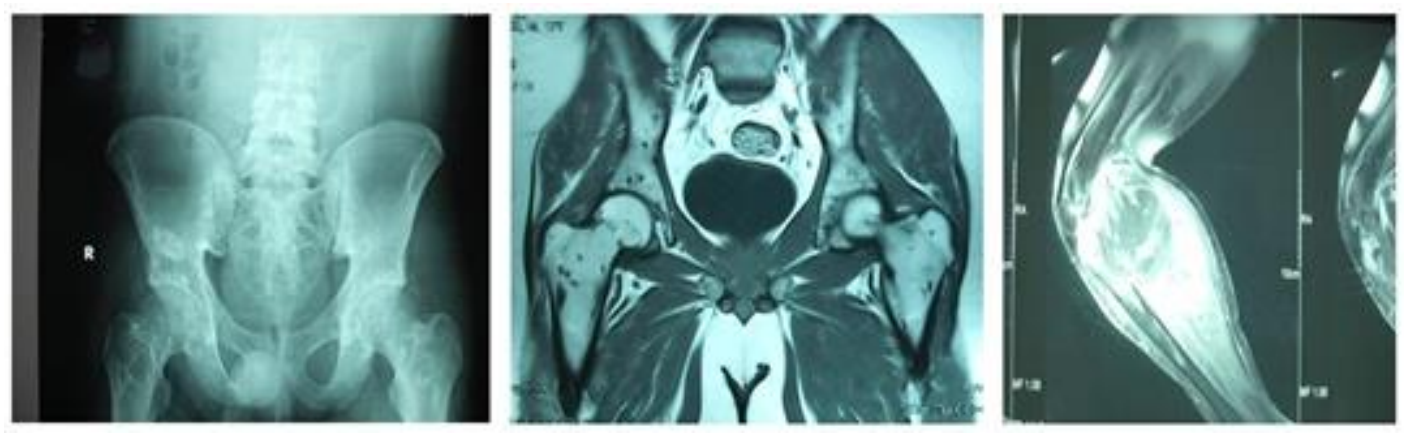

Figure 5 Plain X-ray and MRI right leg showing right proximal tibia osteosarcoma in a 17-year-old boy.

(Abbreviations: MRI: Magnetic resonance imaging).

\subsubsection{Case 5: Cockayne syndrome}

A patient reported a 2 month history of left leg swelling following trauma. He was followed at Al Salt Hospital in Jordan where a hematoma was suspected, and received oral antibiotics showing no improvement. After unsuccessful needle decompression, open decompression and biopsy were performed. Bone scan revealed active lesion proximal to left tibia and chest CT exhibited numerous lung lesions $(1-3 \mathrm{~cm})$ representing metastasis. MRI analysis indicated that there was a large tumor originating from metaphysis of the left tibia (15 cm intramedullary extension) with extraosseous component reaching lateral condyles of femur. The patient progressed and died shortly after 1 month of diagnosis before receiving chemotherapy.

\subsubsection{Case 6: Diamond-BlackfanAnemia}

A 15-year-old girl was diagnosed with DBA and was on regular blood transfusion. She presented at KHCC with osteosarcoma of the right tibia and lung metastasis. The patient needed amputation secondary to osteomyelitis at the 
Abdulqader and Taleb / World Journal of Advanced Research and Reviews, 2020, 06(02), 152-162

site of biopsy taken outside. Therapy consisted of chemotherapy with cisplatinum + doxorubicin, ifosfamide + VP16, HD MTX). She progressed and died after 2.4 years with multiple lung metastases.

\subsection{Literature review}

Research related to osteosarcoma has significantly improved the outcome of treatments and survival rates; however, poor response to treatments worsens the prognosis. There is still much unknown about pathogenesis of the disease and efforts are being directed to discover new targets and develop new treatments.

\subsubsection{Molecular pathogenesis}

Osteosarcoma shows a complex karyotype that may exhibit mutations, translocations, copy-number alterations, and even aneuploidy [7]. These genomic alterations cause lack of genome stability; however, the mechanism by which instability occurs is unknown. Previous studies have suggested that chromothripsis, rupture and reassembly of one or more chromosomes, might be involved, and it has been observed in nearly $90 \%$ of osteosarcomas [3]. Research for driver genes is difficult given that to the date no benign precursor of osteosarcoma has been described, which would help describe tumorigenesis mechanism involved in this type of cancer [8].It has been shown, by next-generation sequencing, that altered genes involved are related to genomic stability maintenance like TP53, RB1, CDK4, MDM2, and ATRX. RB1 is involved in preventing entrance to $\mathrm{S}$ phase of the cell cycle and its mutations lead to cell division. RB1 alterations have been shown in approximately $29-47 \%$ of osteosarcomas $[9,10]$. TP53 have been detected in about 50 90\% of osteosarcomas [8]. CDHK4 and MDM2 are p53 and Rb activity regulators, and are usually overexpressed in osteosarcoma. MDM2 regulates ubiquitination of p53 that leads the protein to degradation [11]. The high relevance of these genes in cell biology prevents their use as treatment targets for osteosarcoma.

\subsubsection{Osteosarcoma and Rothmund-Thomson Syndrome}

RTS is a recessive autosomal genetic disorder caused by a mutation in RECQL 4 that encodes for DNA helicase [12]. Children with RTS present a characteristic rash that evolves to poikiloderma and other dermatological, as well as skeletal abnormalities. Patients with RTS present high risk for developing cancer, particularly osteosarcoma [13]. It has been indicated that $30 \%$ of RTS patients develop osteosarcoma [14]. A previous review showed that $62 \%$ of RTS patients developed osteosarcoma and a cohort study indicated that $32 \%$ developed such cancer $[14,15]$.

\subsubsection{Osteosarcoma and Osteogenesis Imperfecta}

OI is a genetic disorder that causes bone fragility. Four types of OI have been described according to the inheritance, manifestations, and prognosis [16]. Type I is the main type of OI: is an autosomal dominant disease and causes hearing loss, skeletal deformities, and bone fragility among other manifestations. Type II is autosomal recessive and a deadly form of the disease. Types III and IV have normal sclera, unlike the other types, while type IV is a less severe form of the disease.

These patients can present hyperplastic callus that may be similar to osteosarcoma and sometimes be mistaken [17]. Osteosarcoma presentation in patients with OI is rare, nearly 10 cases have been reported to the date $[18,19]$. Early in 1978 only 5 cases had been diagnosed with osteosarcoma and OI [20]. A 14-year-old boy with OI type I exhibited osteosarcoma. Given the similarity of osteosarcoma with hyperplastic callus, his biopsy was delayed, which is necessary for definitive diagnosis [18]. Maiya et al. [19] showed that an early diagnosis of osteosarcoma in OI patients is critical to improve prognosis, and they reported a 6year survival in a type I OI patient of 4 years that received early diagnosis and chemotherapy. It has been proposed that osteosarcoma complication in OI patients might be unrelated to the disease [21]. In contrast, it has been suggested that some factors like frequent fractures, radiation exposure, and metal implants might increase the incidence of osteosarcoma in OI patients [19]. However, the low incidence of both disorders makes the correlation difficult. It must be noticed that in almost all cases diagnosis was delayed and presented a low survival.

\subsubsection{Osteosarcoma and Diamond-Blackfan Anemia}

DBA is a congenital hematopoietic disorder that causes red blood cells aplasia, predominantly during childhood [22]. In 2004, 400 DBA cases had been described worldwide and a higher risk for presenting osteosarcoma has been suggested in these types of patients. The first case of osteosarcoma in DBA patients was described in 1996 by Aquino and Buchanan [23], and an association between the entities was proposed at young age. Since then, approximately 10 cases have been described of osteosarcoma in DBA patients. A study performed on 354 DBA patients suggested that individuals with DBA would have a higher risk for osteosarcoma [22]. 
Mean survival of DBA patients with osteosarcoma was reported as 1.6 years and involved causes like pulmonary metastasis and sepsis. Lee et al. [24] showed that the mean age of presentation for osteosarcoma in DBA patients was between 11 and 12 years old, and that the patients were transfusion dependent. Importantly, the authors reported that 3 patients that developed osteosarcoma had received growth hormone therapy.

Another study observed that a patient with DBA that received bone marrow transplantation at the age of 13 years developed osteosarcoma after 117 months [25]. In this study, the authors associated risk for osteosarcoma after bone marrow transplantation due to different hematopoietic disorders, as the observed 3 cases, and found 7 more cases reported in the literature. They proposed to include osteosarcoma consideration during follow up after bone marrow transplantation and reported favorable prognosis for osteosarcoma presentation in these cases. Furthermore, a clinical trial is being performed to evaluate the risk for osteosarcoma in DBA patients.

\section{Discussion}

A total of 69 patients were diagnosed to have osteosarcoma during the studied period and 6 of them presented syndromes, constituting $8.7 \%$ of all cases. Among children, osteosarcoma represents $2.4 \%$ of cancers [3]. The incidence of osteosarcoma in patients with syndromes is not well established and has been reported to be around $31 \%$ or even as high as 62\% [26]. In general, the incidences reported in syndromic patients were always higher than for sporadic osteosarcoma in children population. To the best of our knowledge, this is the first case series of osteosarcoma and overall predisposing syndromes.

We observed that among non-syndromic patients, 35\% presented metastasis and $30.6 \%$ died. From the syndromic patients, $66.6 \%$ developed metastasis and $80 \%$ died. It has been shown that survival rate in patients with osteosarcoma and metastasis is $30 \%$ and that the rate lowers to $15 \%$ when osteosarcoma relapses [27]. Our results indicated that metastasis was higher among syndromic patients $(\mathrm{p}>0.05, \mathrm{OR}=3.7)$. The high $\mathrm{OR}$ indicates a possible association between metastasis and syndromic patients; however, no statistical significance was reached. It could be considered that the low number of patients analysed could contribute to the absence of statistical significance. Regarding survival, a significantly higher mortality rate was associated to syndromic patients $(\mathrm{p}<0.05,0 \mathrm{R}=9.1)$. It could be hypothesized that syndromic patients present higher risk for presenting osteosarcoma and metastasis. Metastasis has been proven to significantly lower survival rate, which might explain the higher death rate among syndromic patients, suggesting a poorer prognosis among syndromic patients respect to non-syndromic ones [27]. Our results confirm previous reports that showed that syndromic patients have previously been associated to higher cancer and death rates [1].

Likewise, our results indicated that 2 syndromic patients (nearly 30\%) that developed osteosarcoma were RTS patients. The association between RTS and osteosarcoma has been previously reported [28]. A previous study developed by Wang et al. [14] indicated that the incidence of osteosarcoma was 31\% among 41 RTS patients. Truncating mutations of RECQL4 that affect DNA helicase structure have been related to a higher risk to develop osteosarcoma in young patients [29]. This could explain the variability of presentation and outcome between different RTS patients. In fact, one of the patients died after 1.3 years as the result of osteosarcoma relapse and lung metastasis, while the other patient needed chemotherapy and amputation, but survived 10 years and is still alive. In previous reports, it has been shown that nearly half of the patients were long term survivors and the authors proposed that metastasis at diagnosis might be related to their low prognosis results [30]. On the other hand, Hicks et al. [31] indicated that almost $67 \%$ of the patients survived with standard treatment for osteosarcoma.

The age of diagnosis of osteosarcoma on both RTS patients was 9 and 11 years, which is similar to the previously reported mean ages of presentation of 13 years by Zils et al. [30] and 10 years by Hicks et al. [31]. Interestingly, it should be noticed that the age reported in our clinical series was lower than the reported mean age for sporadic osteosarcoma of 15 years [30].

There is still little information about RTS and osteosarcoma and nearly 50 cases have been reported in the literature [30]. Similar to our results, Hicks et al. [31] showed that none of the 12 RTS patients with osteosarcoma presented metastasis at diagnosis, as only one of our patients developed lung metastasis that was absent at the time of osteosarcoma diagnosis. Neither of our patients presented metastasis at osteosarcoma diagnosis.

One of the syndromic cases with osteosarcoma was a 15-year-old female with OI. Regrettably, her follow up was lost after one year. Although rare, there have been some reports in the literature that have suggested a higher risk for osteosarcoma among OI patients [19]. The outcome of osteosarcoma in these types of patients has been suggested to be aggravated by the fact that similarity with hyperplastic callus and its high frequency can delay osteosarcoma diagnosis worsening prognosis [18]. Furthermore, the patient presented history of multiple fractures that has been proposed as 
a predisposing factor for OI patient's presentation of osteosarcoma [19]. The low frequency of both disorders also makes its study difficult and, with only almost 10 cases reported, it must be highlighted that more studies are needed to increase awareness among physicians about the importance of differential diagnosis between hyperplastic callus and osteosarcoma in OI patients.

As far as we know, this is the first case of OI patient that presented osteosarcoma in her forearm. Femur localization was the most frequently reported [19]. Also, the patient survived at least one year without metastasis presentation at diagnosis, which is a little longer than the majority of the cases reported who did not survive through the first year after diagnosis. Remarkably, Maiya et al. [19] indicated that their patient survived 6 years after diagnosis and the authors suggested that awareness and early diagnosis were key to this outcome.

In our study we have identified one case of OPK with osteosarcoma. The patient was a 17year- old boy. OPK is a rare autosomal dominant genetic disorder that causes osteosclerotic dysplasia. The pathogenesis of OPK is unknown and usually causes bone trabeculation with bone islands formation in bones. It presents in 1 of 50,000 cases and is more common in men [32,33]. Generally, OPK is asymptomatic but in some cases it can present with clinical manifestations, such as cutaneous lesions, musculoskeletal disorders, organ abnormalities, endocrine dysfunction, and dental and facial anomalies [34]. Our patient presented clinical features at the age of 2 years, such as growth retardation, mild mental retardation, and hearing loss with erythematous skin rash and scaling and bleeding following sun exposure.

It has been proposed that OPK patients may develop osteosarcoma, as reported by Mindell et al. in their case report performed in 1978 [35]. This was the only case reported in the literature with osteosarcoma presented in an adult patient. The patient was a man of 48 years old with 7 month history of pain and swelling in his left knee. OPK was diagnosed by the presence of bone islands in his ribs, long bones, and small bones of hands and feet. Additionally, biopsy confirmed the presence of osteosarcoma in his left tibia. The patient received chemotherapy and amputation, and progressed to lung metastasis. However, the follow up indicated at least a 5 years survival. Our case report patient presented osteosarcoma in the same localization and referred pain during the previous 4 months. Nonetheless, our patient progressed to lung metastasis and died after 1.8 years. Kucukcakir et al. [34] suggested that OPK patients should be followed up since it has been proposed that increased osteogenesis might be related to osteosarcoma development [36], and follow up of bone activity might help to early diagnosis of osteosarcoma. It could be thought that a follow up of our patient since the age of 2 years and an early diagnosis of OPK might have contributed to an earlier diagnosis of osteosarcoma. Furthermore, it has been reported that OPK lesions in children might increase along with growth and skeleton maturation, perhaps worsening the prognosis of our patient [35].

A patient with CS was diagnosed with osteosarcoma in our study. He presented lung metastasis at diagnosis and died 1 month after. CS is a rare genetic disease characterized by microcephaly, short size, and delayed development [37]. To our knowledge, this is the first case report of CS and osteosarcoma. Although the pathogenesis mechanism involves mutations that affect DNA repair mechanisms, the disease has not been associated to higher risk for cancer [38]. Further studies should be addressed to determine whether it is a case of sporadic osteosarcoma or if there is an association with this disease.

A patient with DBA was also diagnosed with osteosarcoma. The patient presented lung metastasis at diagnosis. Few cases in the world have been diagnosed with DBA and osteosarcoma [22]. As mentioned earlier, Lipton et al. [22] suggested that DBA patients would present a higher risk for osteosarcoma.

The age of presentation (15 years) was a little higher than the previously reported (11-12 years) [24]. On the other hand, the patient survived for 2.4 years after chemotherapy and amputation that was also a little higher than the reported mean survival of 1.6 years [24]. The patient died because of progression to multiple lung metastases, which has previously been described as a cause of poor prognosis not only in DBA patients [24], but also in sporadic osteosarcoma patients [39].

According to our study, $8.7 \%$ of the osteosarcoma patients presented underlying predisposing syndromic features, suggesting that these patients should be suspected of osteosarcoma during differential diagnosis. Even more, although few patients were analyzed, our study might suggest that osteosarcoma presentation in patients with syndromes carries a bad prognosis, as observed by our high death and metastasis rates among syndromic patients (Table 2). 
Table 2 Summary of the reported cases in our clinical series.

\begin{tabular}{|c|c|c|c|c|}
\hline Syndrome & $\begin{array}{l}\text { Age at diagnosis } \\
\text { (years) }\end{array}$ & Bone & $\begin{array}{ll}\text { Type } & \text { of } \\
\text { management }\end{array}$ & Survival \\
\hline CS & 16 & Tibia & None & $\begin{array}{l}\text { Progressed fast and died after } 1 \text { month, } \\
\text { before receiving chemotherapy }\end{array}$ \\
\hline RTS & 9 & Femur & $\begin{array}{l}\text { Chemotherapy and } \\
\text { amputation }\end{array}$ & Alive after 10 years \\
\hline DBA & 15 & Tibia & - & Progressed in lung and died after 2.4 years \\
\hline OI & 14 & Tibia & $\begin{array}{l}\text { Chemotherapy and } \\
\text { amputation }\end{array}$ & Follow up was lost after 1 year \\
\hline RTS & 11 & Femur & Not recorded & $\begin{array}{l}\text { Progressed in lung and local relapse; death } \\
\text { after } 1.3 \text { years }\end{array}$ \\
\hline OPK & 17 & Tibia & Not recorded & $\begin{array}{l}\text { Lung relapse and progression; died after } 1.8 \\
\text { years }\end{array}$ \\
\hline
\end{tabular}

Abbreviations: CS: Cockayne syndrome; DBA: Diamond-Blackfan Anemia; OI: Osteogenesis Imperfecta; OPK: Osteopoikilosis; RTS: RothmundThomson syndrome.

Additionally, considering that some of these syndromes may be accompanied by low stature and delayed growth, growth hormone therapy should be considered as a potential predisposing factor for osteosarcoma in syndromic patients [24]. Moreover, bone marrow transplantation in DBA patients should also be considered carefully given the previous reports that suggest an increased risk for osteosarcoma [25].Although previous studies have reported a potential association between most of the syndromes indicated in our work and osteosarcoma, this is the first case of a CS as well as OPK patients that presented osteosarcoma.

\section{Conclusion}

Previous research studies have reported a potential association between most of the syndromes indicated in our work and osteosarcoma, this is the first case of a CS as well as OPK patients that presented osteosarcoma. This study revealed that osteosarcoma carried bad prognosis when associated with syndromes, although the number is small. High index of suspicion to look for syndromic features in each OS Patient is warranted. Further research studies are needed to be carried out in CS and OPK patients to confirm these observations.

\section{Limitations of the study}

The main limitations of this study include the low number of patients analyzed that is related to the low frequency of presentation of the diseases studied, including osteosarcoma per se. The comparison between the percentages of metastasis in non-syndromic patients vs. syndromic ones did not show a statistical significance, and more patients should be considered to reach trustworthy results. Moreover, each syndrome has its own particular characteristics and its relation to osteosarcoma would probably be unique, suggesting that it would be appropriate to continue the study by considering each syndrome separately.

The other limitation is related to the fact that none of the syndromic genes have been documented due to lack of genomic analysis.

\section{Compliance with ethical standards}

\section{Acknowledgments}

We would like to offer a special thanks to our colleagues and staff at King Hussein Cancer Center (KHCC) who provided a great cooperation. Our special thanks are extended to Dr. Yakub Sayyad who made language editing service.

\section{Disclosure of conflict of interest}

Author collected patient data and prepared the manuscript, and evaluated data, and submitted the manuscript. All the authors are guarantor for this manuscript. There is no any conflict of interest among authors. Author affirms that this 
manuscript is an honest, accurate, and transparent account of the study being reported; that no important aspects of the study have been omitted.

\section{Statement of ethical approval}

Ethical approval (No.12 KHCC 64) was obtained from Institutional Board Review (IRB) at King Hussein Cancer Center in Jordan.

\section{Statement of informed consent}

Informed consent was obtained from all individual participants included in the study.

\section{References}

[1] Hameed M and Mandelker D. (2018). umor syndromes predisposing to osteosarcoma. AdvAnatPathol, 25(4), 217-22.

[2] Prater S and McKeon B. (2019). Cancer, osteosarcoma. In: StatPearls, editor [Internet]. Treasure Island (FL): StatPearls Publishing.

[3] Ottaviani G and Jaffe N. (2009). The epidemiology of osteosarcoma. In: Jaffe N, Bruland OS, Bielack S, editors. Pediatric and Adolescent Osteosarcoma [Internet]. Boston, MA: Springer US, 3-13.

[4] Jawad MU and Scully SP. (2010). In brief: classifications in brief: enneking classification: benign and malignant tumors of the musculoskeletal system. Clin Orthop Relat Res, 468(7), 2000-2.

[5] Tanaka K and Tsumura H. (2019). Eighth edition of the American Joint Committee on Cancer staging system for soft tissue sarcoma of the trunk and extremity: in search of a better staging system. Ann Transl Med, 7(S1), S11.

[6] Wadhwa N. (2014). Osteosarcoma: Diagnostic dilemmas in histopathology and prognostic factors. Indian J Orthop, 48(3), 247-54.

[7] Chen X, Bahrami A, Pappo A, Easton J, Dalton J, Hedlund E, etal. (2014). Recurrent somatic structural variations contribute to tumorigenesis in pediatric osteosarcoma. Cell Reports, 7(1), $104-12$.

[8] Franceschini N, Lam SW, Cleton-Jansen A-M and Bovée JVMG. (2020).What's new in bone forming tumours of the skeleton? Virchows Arch, 476(1), 147-57.

[9] Kovac M, Blattmann C, Ribi S, Smida J, Mueller NS, Engert F, etal. (2015). Exome sequencing of osteosarcoma reveals mutation signatures reminiscent of BRCA deficiency. Nat Commun, 6(1), 8940.

[10] Fittall MW, Mifsud W, Pillay N, Ye H, Strobl AC, Verfaillie A, etal. (2018). Recurrent rearrangements of FOS and FOSB define osteoblastoma. Nat Commun, 9(1), 2150.

[11] Freedman D, Wu L and Levine A. (1999). Functions of the MDM2 oncoprotein. Cell Mol Life Sci, 55(1), 96-107.

[12] Maciaszek JL, Oak N, Chen W, Hamilton KV, McGee RB, Nuccio R, etal. (2019). Enrichment of heterozygous germline RECQL4 loss-of-function variants in pediatric osteosarcoma. Cold Spring HarbMol Case Stud, 5(5), a004218.

[13] Wang L, Gannavarapu A, Kozinetz C, Levy M, Lewis R, Chintagumpala M, etal. (2003). Association between osteosarcoma and deleterious mutations in the RECQL4 gene in Rothmund-Thomson syndrome. J Natl Cancer Inst, 95(9), 669-74.

[14] Wang LL, Levy ML, Lewis RA, Chintagumpala MM, Lev D, Rogers M, et al. (2001). Clinical manifestations in a cohort of 41 Rothmund-Thomson syndrome patients.Am J Med Genet, 102(1), 11-7.

[15] Stinco G, Governatori G, Mattighello P and Patrone P. (2008). Multiple cutaneous neoplasms in a patient with Rothmund-Thomson syndrome: Case report and published work review. J Dermatol, 35(3), 154-61.

[16] Sillence D. (1981). Osteogenesis imperfecta: an expanding panorama of variants. ClinOrthop, 159, 11-25.

[17] Ertze J and Lennartz K. (1969). Callus luxuriansbei Osteogenesis imperfecta unterdemBildeeinesSarkoms. Z Orthop, 106, 463-75.

[18] Reid BS and Hubbard JD. (1979). Osteosarcoma arising in osteogenesis imperfecta. PediatrRadiol, 8(2), 110-2. 
[19] Maiya S, Grimer R, Ramaswamy R and Deshmukh N. (2002). Osteosarcoma occurring in osteogenesis imperfecta tarda. IntOrthopaed, 26(2), 126-8.

[20] Lasson U, Harms D and Wiedemann HR. (1978). Osteogenic sarcoma complicating osteogenesis imperfecta tarda. Eur J Pediatr, 129(3), 215-8.

[21] Gagliardi JA, Evans EM, ChandnaniVP, Myers JB and Pacheco CM. (1995). Osteogenesis imperfecta complicated by osteosarcoma. Skeletal Radiol, 24(4), 308-10.

[22] Lipton JM, Federman N, Khabbaze Y, Schwartz CL, Hilliard LM, Clark JI, etal. (2001). Osteogenic sarcoma associated with Diamond Blackfan anemia: a report from the diamond Blackfan anemia registry. J Ped Hematol Oncol, 23(1), 39-44.

[23] Aquino VM and Buchanan GR. (1996). Osteogenic sarcoma in a child with transfusion-dependent diamondblackfan anemia. J Ped Hematol Oncol, 18(2), 230-2.

[24] Lee RS, Higgs D, Haddo O, Pringle J and Briggs TWR. (2004). Osteosarcoma associated with diamond-blackfan anaemia: a case of a child receiving growth hormone therapy. Sarcoma, 8(1), 47-9.

[25] Ueki H, Maeda N, Sekimizu M, Tsukushi S, Nishida Y and Horibe K. (2013). Osteosarcoma after bone marrow transplantation. J Ped Hematol Oncol, 35(2), 134-8.

[26] Calvert GT, Randall RL, Jones KB, Cannon-Albright L, Lessnick S and Schiffman JD. (2012). At-risk populations for osteosarcoma: the syndromes and beyond. Sarcoma, 152382.

[27] Anninga JK, Gelderblom H, Fiocco M, Kroep JR, Taminiau AHM, Hogendoorn PCW, etal. (2011). hemotherapeutic adjuvant treatment for osteosarcoma: Where do we stand? Eur J Cancer, 47(16), 2431-45.

[28] Lu L, Jin W, Liu H and Wang LL. (2014). RECQ DNA helicases and osteosarcoma.Adv Med Biol, 804, 129-45.

[29] Colombo E, Locatelli A, Cubells Sánchez L, Romeo S, Elcioglu N, Maystadt I, etal. (2018). Rothmund-thomson syndrome: insights from new patients on the genetic variability underpinning clinical presentation and cancer outcome. IntJMolSci, 19(4), 1103.

[30] Zils K, Klingebiel T, Behnisch W, Mueller HL, Schlegel PG, Fruehwald M, etal. (2015). Osteosarcoma in patients with rothmund-thomson syndrome. Ped Hematol Oncol, 32(1), 32-40.

[31] Hicks MJ, Roth JR, Kozinetz CA and Wang LL. (2007). Clinicopathologic features of osteosarcoma in patients with rothmund-thomson syndrome. J Clin Oncol, 25(4), 370-5.

[32] Resnick D, Niwayama G, Enostosis, hyperostosis and periostitis. (1995). In:Resnick D, Niwayama D, editors. Diagnosis of Bone and Joint Disorders. Philadelphia:W.B Saunders Company, 4404-11.

[33] Benli I, Akalin S, Boysan E, MumcuE,Kiş M and Türkoğlu D. (1992). Epidemiological, clinical and radiological aspects of osteopoikilosis. J Bone Joint Surg Br, 74(4), 504-6.

[34] Kucukcakir N, AltanInceoglu L and LatifRaif S. (2015). Osteopoikilosis-a case report. Turk J Phys Med Rehab. 61(4), 375-9.

[35] Mindell E, Northup C and Douglass HJ. (1978). Osteosarcoma associated with osteopoikilosis. J Bone Joint Surg Am, 60(3), 406-8.

[36] Dahlin DC. (1975). Pathology of Osteosarcoma. ClinOrthop, 111, 23-32.

[37] Genetics Home Reference. (2020). Cockayne syndrome [Internet]. Genetics Home Reference.

[38] Reid-Bayliss KS, Arron ST, Loeb LA, Bezrookove V and Cleaver JE. (2016). Why Cockayne syndrome patients do not get cancer despite their DNA repair deficiency. Proc Natl Acad Sci USA, 113(36), 10151-6.

[39] Huang X, Zhao J, Bai J, Shen H, Zhang B, Deng L, etal. (2019). Risk and clinicopathological features of osteosarcoma metastasis to the lung: A population-based study. J Bone Oncol, 16, 100230.

\section{How to cite this article}

Al-Hebshi, A. and Ismael, T. (2020). Syndromic osteosarcoma, does it carry a poor prognosis? King Hussein Cancer Center experience. A case series report with literature review. World Journal of Advanced Research and Reviews, 6(2), 152-162. 